
\title{
25 Research Square \\ Synthetic MRI for Stroke: A Qualitative and Quantitative Pilot Study
}

Joachim André ( $\square$ joachim.andre@ulb.be )

Erasmus Hospital

Sami Barrit

Erasmus Hospital

Patrice Jissendi Tchofo

Centre Hospitalier Universitaire de Saint-Pierre

\section{Research Article}

Keywords: Synthetic MRI, brain, stroke, qualitative analysis, quantitative MRI, relaxometry

Posted Date: January 10th, 2022

DOI: https://doi.org/10.21203/rs.3.rs-1184348/v1

License: (c) (i) This work is licensed under a Creative Commons Attribution 4.0 International License. Read Full License 


\section{Abstract}

\section{Purpose}

Synthetic MR provides quantitative multiparametric data about tissue properties in a fast singleacquisition. We compared synthetic and conventional image quality and investigated synthetic relaxometry of acute and chronic ischemic lesions to support its interest in stroke imaging.

\section{Methods}

For this pilot study, we prospectively acquired synthetic and conventional brain MR of 43 consecutive adult patients with suspected stroke. We studied a total of 136 lesions, of which 46 DWI-positive with restricted ADC (DWI+/rADC), 90 white matter T2/FLAIR hyperintensities $(\mathrm{WMH})$, and 430 normal brain regions (NBR). We assessed image quality for lesion definition according to a 3-level score by two readers of different experiences. We compared relaxometry of lesions and regions of interest.

Results

Synthetic images were superior to their paired conventional images for lesion definition except for sFLAIR (sT1 or sPSIR vs. cT1 and sT2 vs. cT2 for DWI+/rADC and WMH definition; p-values <.001) with substantial to almost perfect inter-rater reliability ( $\mathrm{K}$ ranging from 0.711 to 0.932 , $\mathrm{p}$-values $<.001$ ). We found significant differences in relaxometry between lesions and NBR and between acute and chronic lesions (T1, T2, and PD of DWI+/rADC or WMH vs. mirror NBR; $p$-values $<.001 ; T 1$ and PD of DWI+/rADC vs. WMH; p-values of 0.034 and 0.008$)$.

\section{Conclusion}

Synthetic MR may contribute to stroke imaging by fast acquiring consistent relaxometry data and accessible derived images of interest for the study of ischemic lesions.

\section{Introduction}

Stroke care relies on timely and proper imaging. In the acute stage, it guides therapeutic interventions such as intravenous thrombolysis, endovascular treatments, and neurosurgery by early detection of acute ischemic stroke and intracranial hemorrhage $(\mathrm{ICH})$ and characterization of infarct core penumbra and cerebral circulation. In the subacute or chronic stages, it supports the clinician's diagnosis, management, and prognosis by describing lesion evolution and associated brain abnormalities. As MRI performance for these purposes is well substantiated, its scanning duration remains a drawback. [1, 2] Along these lines, emerging techniques such as synthetic MR imaging (sMRI) are promising by their potential to provide fast single-acquisition of quantitative multiparametric data about tissue properties using a multi-dynamic multi-echo sequence. This sequence repeats the same gradient reversal process used to create a single gradient-echo to produce additional gradient-echoes after a unique radiofrequency pulse. [3, 4] Synthetic weighted images may be generated by virtually adjusting parameters such as echo, repetition, and 
inversion delay times, using mathematical inferences rather than being predetermined by multiple acquisitions of different sequences as in conventional MR imaging (cMRI). Different uses of sMRI in neuroimaging were reported for gliomas, brain metastasis, and multiple sclerosis. [5-9] To our knowledge, only two recent studies investigated SMRI application for stroke. Duchaussnoy et al. reported the specific interest in synthetic T2 mapping to gauge wake-up stroke onset time. [10] Li et al. provided a phantom validation study of the reliability of sMRI relaxometry measures for chronic ischemic stroke. [11] Hence, no study has specifically addressed overall sMRI clinical applicability for stroke. Therefore, we compared synthetic and conventional paired images and investigated synthetic relaxometry for acute and chronic ischemic lesions in controlled clinical conditions.

\section{Methods}

\subsection{Study population:}

The institutional review board (Comité d' éthique du CHU Saint-Pierre) approved the study and waived the need for informed consent. This study was performed in accordance with relevant guidelines and regulations. We prospectively enrolled a total of 45 consecutive adult patients (aged 18 or more) admitted for sudden onset of neurological deficit or symptoms highly suggestive of stroke from January 2017 to March 2018. Our inclusion criteria were (i) conclusion of the early management (i.e. at least 24 hours after their admission) with (ii) no therapeutic interventions (i.e., intravenous fibrinolysis, endovascular treatment, or neurosurgery) (iii) no acute ICH diagnosed. We performed a dedicated MRI session comprising conventional and synthetic acquisitions for this purpose. We observed four technical incidents: two patients presented major motion artifacts hindering conventional images interpretation, and two did not undergo the synthetic acquisition due to human operator error. A diagram illustrates the flow of participants through the study (Fig S1. in Supplemental Material).

\subsection{MRI acquisition:}

The whole patient group was scanned on a 1.5T MR imaging scanner (GE Healthcare, Chicago, IL) using a 24-channel head coil. Institutional cMRI stroke protocol includes 2D axial T1, FLAIR, T2*, DWI and apparent diffusion coefficient (ADC) mapping, coronal PROPELLER T2, and 3D TOF sequences. A 2D axial multi-dynamic multi-echo acquisition was then performed to provide the quantitative mapping of $\mathrm{T} 1$, T2, and PD using MAGnetic resonance image Compilation (MAGiC, GE Healthcare) ${ }^{5}$ post-processing software. Synthetic images generated by MAGiC based on longitudinal $R_{1}$ relaxation rate, transverse $R_{2}$ relaxation rate, and PD maps have been adapted to maximize tissue contrast. The total protocol duration was $24 \mathrm{~min}$ (17 $\mathrm{min} 20 \mathrm{sec}$ for the conventional protocol and $6 \mathrm{~min} 40 \mathrm{sec}$ for the synthetic acquisition).

\subsection{Image analysis:}

DWI-positive with restricted ADC (DWI+/rADC) or white matter T2/FLAIR hyperintensities not meeting the previous criteria (WMH) were defined based on the cMRI images (Fig. 1) by consensus between an 
experienced neuroradiologist (PJ) and a neuroradiologist trainee (JA). For this purpose, they were referred to as acute or chronic ischemic lesions, respectively.

\subsubsection{Qualitative analysis:}

We performed the qualitative analysis for the first 25 consecutive eligible patients. Images were independently, randomly, and blindly assessed after a minimal 2-week memory-washout period of the consensus mentioned above on PACS consoles using the same settings for image display. We collected the qualitative assessment of lesions visibility and definition using a data collection sheet according to a 3-level score for each sequence of cMRI (cT1, cT2, cFLAIR) and sMRI (sT1, sPSIR, sT2, sFLAIR) as 1 (no lesion visible), 2 (lesion visible), 3 (well-defined lesion).

\subsubsection{Quantitative analysis:}

A single investigator (JA) performed the quantitative data collection on MAGiC on the 43 eligible patients. We delineated a circular ROI of DWI+/rADC and WMH lesions aiming to maximize the ROI-lesion overlap while not including perilesional tissue on the synthetic images based on a visual matching with the $\mathrm{cDWl}$ and cFLAIR images. Then, we placed a circular ROI of the same size in the contralateral apparent normal brain region (NBR) for each lesion in the same section and location, so-called the mirror ROI (Fig. 2). For $\mathrm{WMH}$, we considered only the most representative lesion - i.e., the most extensive lesion presenting a homogeneous hypersignal in cFLAIR with an adequate mirror ROI per patient. We manually placed ten ROIs in different NBR structures: superficial and deep white matter (WM), grey matter (GM), and cerebrospinal fluid (CSF). The superficial and deep WM structures corresponded to left and right centrum semiovale, and, genu and splenium of the corpus callosum; the superficial and deep GM structures corresponded to left and right superior frontal cortex and thalami; the CSF structures corresponded to left and right anterior horns of the lateral ventricles. We collected R1, R2, and PD values. The works of Blystad et al. inspired this approach. [3]

\subsection{Statistical analysis.}

We compared paired or independent ordinal variables or dichotomous dependent variables by nonparametric tests of Wilcoxon signed-rank, Mann-Whitney-Wilcoxon, or McNemar's test, respectively. The inter-rater reliability was measured by quadratic Cohen's weighted kappa coefficient ( $\mathrm{K}$ ). We calculated the Kendall rank correlation coefficient $(\tau)$ for the ordinal association. Statistical significance was predetermined as a $\mathrm{p}$-value $<0.05$ with preferred two-tailed tests. We computed statistics and data visualization on the open-source R, Version 4.0.3 (R statistical and computing software; http://www.rproject.org/).

\section{Results}

\subsection{Study population}


The 43 patients included were 16 females (age range 25-84 years, mean age 54 years) and 27 males (age range 22-89 years, mean age 63 years), with a combined total of 136 lesions of which 46 DWI+/rADC and $90 \mathrm{WMH}-4$ patients being free of diagnosed lesions, and, 430 NBR. We did not find any DWI/FLAIR mismatched lesion of potentially undiagnosed silent hyperacute stroke or T2* hypointensity of an acute $\mathrm{ICH}$.

\subsection{Qualitative analysis}

The assessed quality of synthetic images was significantly superior to their paired conventional images for acute and chronic ischemic lesions except for sFLAIR (sT1 or sPSIR vs. cT1 and sT2 vs. cT2 for DWI+/rADC and WMH definition; $p$-values <.001). Indeed, sFLAIR qualitative comparison was only significantly superior for chronic ischemic lesions (sFLAIR vs. cFLAIR for DWI+/rADC and WMH definition, $\mathrm{p}$-values of 0.49 and <.001). The readers reported a higher noise level and a greater number of artifacts (consisting of prototypical hyperintensities) resulting in lower overall image quality of sFLAIR than cFLAIR. The proportion of visible lesions on ST1 and SPSIR was significantly superior for acute and chronic ischemic lesions (sT1 or SPSIR vs. cT1 for DWI+/rADC and WMH visibility, p-values <.001). A grouped and stacked barplot displays these results (Fig. 3). All inter-rater reliability measures were significantly satisfactory, with the lowest observed for cT2 and the highest for cFLAIR $(\mathrm{K}=0.47$ [0.29 0.66 ] and 0.93 [0.89 - 0.98], p-values <.001). Tables display these results (Tables S1 to S3 in Supplemental Material).

\subsection{Quantitative analysis}

All relaxometry measures of acute and chronic ischemic lesions compared to their mirror NBR were significantly different $(T 1, T 2$, and PD of $D W I+/ r A D C$ or $W M H$ vs. mirror NBR; $p$-values <.001). Relaxometry measures of acute compared to chronic ischemic lesions were significantly different except T2 (T1, T2, and PD of DWI+/rADC vs WMH; p-values of $0.034,0.697$, and 0.008 ). Concerning NBR paired relaxometry measures, only the T2 of the right and left frontal cortex, and the genu and splenium of corpus callosum ROls were significantly different ( $p$-values of 0.042 and 0.035 ). Age was rank-correlated with statistical significance for all relaxometry measures of CSF and centrum semiovale, T2 and PD of thalamus and T2 of cortex and genu of corpus callosum (absolute value of $\tau$ ranging from 0.16 to 0.42 , p-values from 0.036 to <.001). A beeswarm-box plot (Fig. 4) and tables display these results (Tables S4 and S5 in Supplemental Material).

\section{Discussion}

Due to scarce evidence available, a full-scale study implementing sMRI in the management of stroke patients from the outset was considered premature. Therefore, this pilot study aimed to investigate the interest of sMRI for ischemic stroke in controlled clinical conditions. Of the 45 consecutive patients enrolled, four were concerned by technical incidents. None were due to the synthetic acquisition but two related to it as human operator errors. Specific training and experience combined with dedicated MRI protocols for clinical application should prevent these. The remaining two technical incidents were major 
motion artifacts only compromising conventional images. We did not observe any adverse events concerning their clinical management.

\subsection{Qualitative analysis:}

The quality of synthetic images was assessed superior to conventional weighted images for $D W 1+/ \mathrm{rADC}$ and WMH definition - sFLAIR not reaching significance, particularly sT1 and SPSIR compared to CT1 for acute and chronic ischemic lesions. The interest of sPSIR has been previously reported for multiple sclerosis lesions detection and intracranial vessel characterization. To our knowledge, no previous study has evaluated its interest in stroke. Substantial to almost perfect inter-rater reliability was obtained for synthetic imaging between the two observers but lower than conventional imaging, except for the cT2. Both readers were naive to SMRI, which may contribute, combined with a significant difference in experience, to the overall lower inter-individual agreement observed for synthetic images. The cT2 lowest inter-rater reliability exception may be due to a coronal acquisition thought to be more difficult to interpret for inexperienced readers. Interestingly, this suggests that the specification of cMRI acquisition protocol may have more effect on inter-reader agreement than the synthetic generation of weighted images. According to the readers' feedback, an sFLAIR lower overall image quality than cFLAIR due to noise and artifacts was observed. However, this subjective assessment was not associated with a significant difference in image quality ratings for lesions definition and visibility on FLAIR images for inter-method (synthetic versus conventional) and inter-readers qualitative assessments (experienced neuroradiologist versus trainee) comparisons. This supports easily detectable artifacts not hindering lesion characterization even for inexperienced readers in line with previous findings of Ryu et al. [12]

\subsection{Quantitative analysis:}

Acute and chronic ischemic lesions relaxometry profiles compared to their mirror NBR showed higher lesional T1, T2, and PD. Interestingly, a comparison between acute versus chronic ischemic lesions profiles retrieved significant differences for T1 and PD but not T2. These two lesion groups presented heterogeneous relaxometry values (as depicted in Figure 4) may be due to high variability in lesion age and topography. Along these lines, Duchaussoy et al. reported a correlation between synthetic T2 mapping and stroke onset time, supporting it as a potential biomarker of interest for the management of patients with stroke of unknown onset. [10] Overall quantitative findings of NBR were in line with the literature, although variations in populations studied and methods of segmentation of ROIs (automated, semi-automated, or manually drawn) and technology (e.g., quantitative cMRI, sMRI, or MRI fingerprinting) to acquire the relaxometry may contribute to differences observed. [13-17] Interestingly, we found a significant difference in T2 relaxometry between left and right frontal cortex ROls. An explanation may be the cortico-subcortical atrophy of the overrepresented elderly patients in the study population, associated with the partial volume effect of CSF uncovering a potential left-right asymmetric segmentation bias due to the manual method used. ${ }^{18}$ In this study, $T 1, T 2$, and PD of WM values increased with age which is consistent with the recent findings of Hagiwara et al. [19]. Our nonsignificant finding for the grey matter's PD decreasing with age may corroborate the previous hypothesis postulated about the potential left-right 
asymmetric segmentation bias due to the manual segmentation method used, partial volume effect, and population studied.

\section{Limits:}

As a pilot study, only selected patients - i.e., after the conclusion of their early management and clinical stability ensured - were recruited in a dedicated MRI session. Our study was not designed to evaluate sMRI diagnosis performance nor its clinical significance. Indeed, we assessed lesions definition and visibility based on a consensus. No receiver operating characteristic analysis could then be performed. Furthermore, its small sample size prevented complementary analyses, such as image quality depending on lesion topography.

\section{Perspectives:}

A full-scale study of sMRI for stroke would be the next step to confirm its clinical applicability and significance. Its diagnosis performance for specific stroke imaging endpoints compared to criterion standards has to be determined in clinical settings comprising the critical time window of acute stroke early management. The sMRI may fastly provide surrogate cMRI images and unique information for stroke care. Indeed, sMRI can offer versatile relaxometry weighting parameters dynamically adjustable for contrast and color-coded visualization. Moreover, the synthetic quantitative data may be particularly suitable for computational analysis to develop lesion automated detection algorithms (e.g., of hyperacute ischemic lesion or ICH), [20] define biomarkers (e.g., of stroke salvageability, onset time, or topography), [10] and, constitute voxel-wise stroke-specific atlases. [21]

\section{Conclusion}

An MRI protocol combining conventional and synthetic acquisitions was performed on 43 consecutive stroke patients in controlled clinical conditions without adverse events due to this technology. The qualitative analysis of synthetic MRI found superior overall performance for acute and chronic ischemic lesions definition compared to conventional MRI. Moreover, the inter-rater reliability between two readers with different experiences supports the accessibility of this technique. The quantitative analysis of synthetic relaxometry found significant differences in profiles of acute and chronic ischemic lesions and consistent findings of distinct normal brain regions, with the literature suggesting promising interest in this approach for stroke. Therefore, a full-scale study of synthetic MRI diagnosis performance in clinical settings comprising the critical time window of acute stroke early management would be the next step to investigate its clinical significance. Moreover, the synthetic quantitative data may define useful stroke biomarkers for future radiomics applications.

\section{Abbreviations}

SMRI = synthetic MR imaging; $c M R I=$ conventional MR imaging; $I C H$ = intracranial hemorrhage; $P D=$ proton density; MAGiC = MAGnetic resonance image Compilation; $\mathrm{DIR}=$ double inversion recovery; PSIR = 
phase-sensitive inversion recovery; NBR = normal brain region; $\mathrm{K}=$ Cohen's kappa coefficient; $\tau=$ Kendall's tau rank correlation coefficient; $\mathrm{pu}=$ percentage unit

\section{Declarations}

Funding: None.

Statements and Declarations: The authors have no conflict of interest nor competing interest to declare. The data and code are fully available upon request for reviewers to test reproducibility. About ethical considerations, the institutional review board approved this study while waiving consent to participate/for publication. We certify that the submission is original work and is not under review at any other publication.

\section{References}

1. Powers W, Rabinstein A, Ackerson T, et al. Guidelines for the early management of patients with acute ischemic stroke: 2019 update to the 2018 guidelines for the early management of acute ischemic stroke: a guideline for healthcare professionals from the American Heart Association/American Stroke Association. Stroke 2019;50:e344-e418.

DOI: https://doi.org/10.1161/STR.0000000000000211

2. Provost C, Soudant M, Legrand L, et al. Magnetic Resonance Imaging or Computed Tomography Before Treatment in Acute Ischemic Stroke. Stroke 2019;50:659-664.

DOI: https://doi.org/10.1161/STROKEAHA.118.023882

3. Blystad I, Warntjes JBM, Smedby O, et al. Synthetic MRI of the brain in a clinical setting. Acta Radiol 2012;53:1158-1163. DOI: https://doi.org/10.1258/ar.2012.120195

4. Hagiwara A, Warntjes JBM, Hori M, et al. SyMRI of the brain: rapid quantification of relaxation rates and proton density, with synthetic mri, automatic brain segmentation, and myelin measurement. Invest Radio/2017;52(10):647-657. DOI: https://doi.org/10.1097/RLI.0000000000000365

5. Tanenbaum L, Tsiouris A, Johnson A, et al. Synthetic MRI for clinical neuroimaging: results of the magnetic resonance image compilation (magic) prospective, multicenter, multireader trial. AJNR Am J Neuroradio/ 2017;38(6):1103-1110. DOI: https://doi.org/10.3174/ajnr.A5227.

6. Blystad I, Warntjes JBM, Larsson M, et al. Quantitative MRI for analysis of peritumoral edema in malignant gliomas. PLoS one 2017;12(5):e0177135.

DOI: https://doi.org/10.1371/journal.pone.0177135

7. Hagiwara A, Hori M, Suzuki M, et al. Contrast-enhanced synthetic MRI for the detection of brain metastases. Acta Radiol Open 2016;15;5(2):2058460115626757.

DOI: https://doi.org/10.1177/2058460115626757

8. Granberg T, Uppman M, Hashim F, et al. Clinical feasibility syntheticMR in multiple sclerosis: a diagnostic and volumetric validation study. AJNR Am J Neuroradiol 2016;37(6):1023-9.

DOI: https://doi.org/10.3174/ajnr.A4665 
9. Hagiwara A, Hori M, Yokoyama $\mathrm{K}$, et al. Synthetic MRI in the detection of multiple sclerosis plaques. Synthetic MRI in the detection of multiple sclerosis plaques. AJNR Am J Neuroradio/ 2017;38(2):257263. DOI: https://doi.org/10.3174/ajnr.A5012

10. Duchaussoy T, Budzik JF, Norberciak L, et al. Synthetic T2 mapping is correlated with time from stroke onset: a future tool in wake-up stroke management? Eur Radiol 2019;29(12):7019-7026. DOI: https://doi.org/10.1007/s00330-019-06270-0

11. Li CW, Hsu AL, Huang CW, et al. Reliability of synthetic brain MRI for assessment of ischemic stroke with phantom validation of a relaxation time determination method. J Clin Med 2020;14;9(6):1857. DOI: https://doi.org/10.3390/jcm9061857

1. Ryu K, Baek H, Moon J, et al. Initial clinical experience of synthetic MRI as a routine neuroimaging protocol in daily practice: A singlecenter study. J Neuroradiol 2020;47(2):151-160. DOI: https://doi.org/10.1016/j.neurad.2019.03.002

13. Deoni S, Peters T, Rutt B. High-resolution T1 and T2 mapping of the brain in a clinically acceptable time with DESPOT1 and DESPOT2. Magn Reson Med 2005;53(1):237-41.

DOI: https://doi.org/10.1002/mrm.20314

14. Warntjes JBM, Leinhard OD, West J, et al. Rapid magnetic resonance quantification on the brain: optimization for clinical usage. Magn Reson Med 2008;60(2):320-9.

DOI: https://doi.org/10.1002/mrm.21635

15. West J, Warntjes JBM, Lundberg P. Novel whole brain segmentation and volume estimation using quantitative MRI. Eur Radio/ 2012;22(5):998-1007. DOI: https://doi.org/10.1007/s00330-011-2336-7

16. Warntjes JBM, Engström $M$, Tisell $A$, et al. Brain characterization using normalized quantitative magnetic resonance imaging. PLoS One 2013;8(8):e70864. DOI:

https://doi.org/10.1371/journal.pone.0070864

17. Körzdörfer G, Kirsch R, Liu K, et al. Reproducibility and repeatability of MR fingerprinting relaxometry in the human brain. Radiology 2019;292(2):429-437. DOI: https://doi.org/10.1148/radiol.2019182360

18. Maltbie $E$, Bhatt $K$, Paniagua $B$, et al. Asymmetric bias in user guided segmentations of brain structures. Neuroimage 2012;59(2):1315-23. DOI: https://doi.org/10.1016/j.neuroimage.2011.08.025

19. Hagiwara A, Fujimoto K, Kamagata K, et al. Age-related changes in relaxation times, proton density, myelin, and tissue volumes in adult brain analyzed by 2-dimensional quantitative synthetic magnetic resonance imaging. Invest Radiol 2021;56(3):163-172. DOI:

https://doi.org/10.1097/RLI.0000000000000720

20. Nael K, Gibson E, Yang C, et al. Automated detection of critical findings in multi-parametric brain MRI using a system of 3D neural networks. Sci Rep 2021;11(1):6876. DOI: 
https://doi.org/10.1038/s41598-021-86022-7

21. Wang Y, Juliano J, Liew SK, et al. Stroke atlas of the brain: Voxel-wise density-based clustering of infarct lesions topographic distribution. Neuroimage Clin 2019;24:101981. DOI:

https://doi.org/10.1016/j.nicl.2019.101981

\section{Figures}

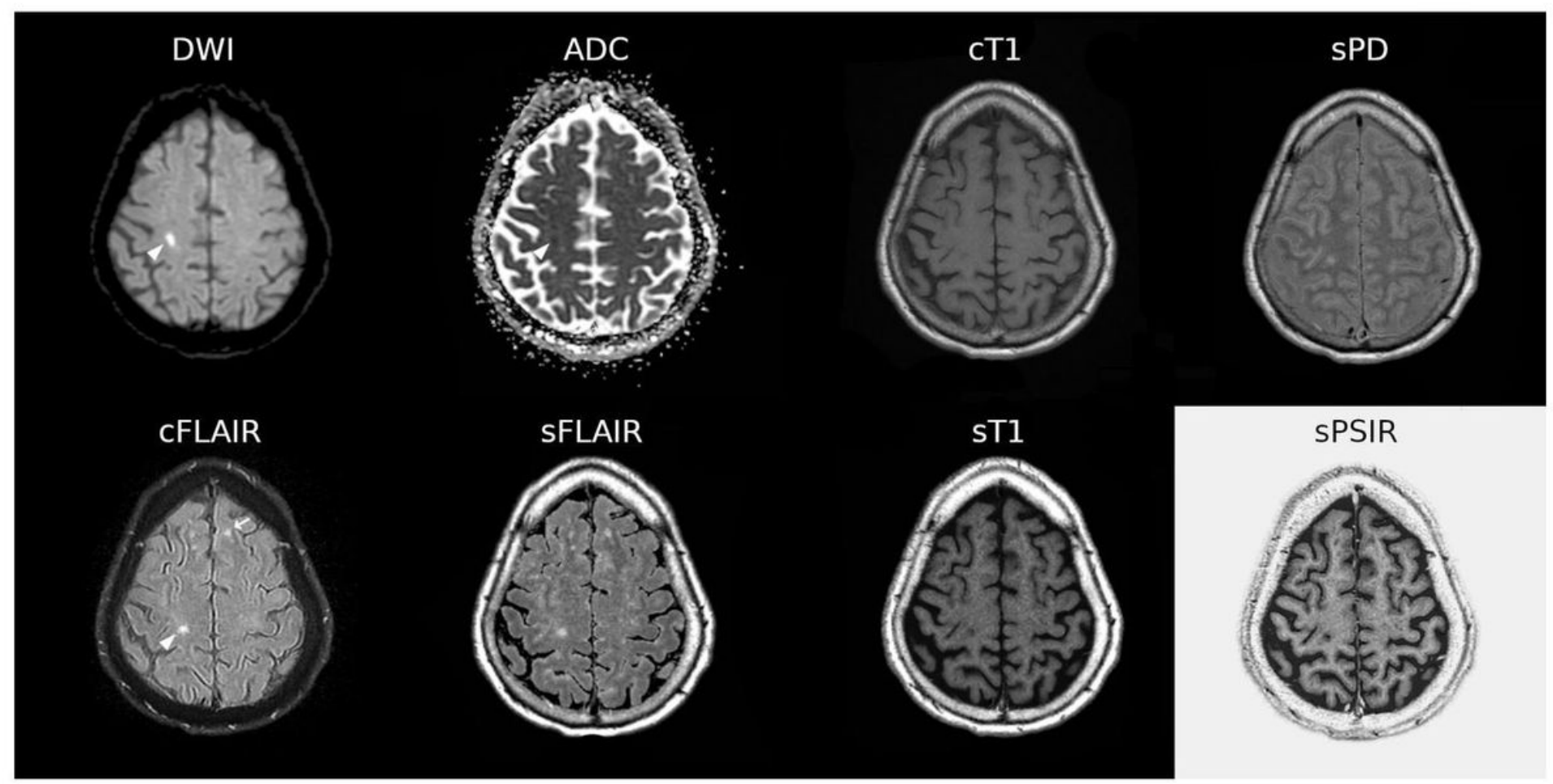

\section{Figure 1}

Axial conventional (DWI, ADC, cFLAIR, cT1) and synthetic (sFLAIR, sT1, sPD, sPSIR) weighted images depicting acute (DWI+/rADC, arrowhead) and chronic (WMH, arrow) ischemic lesions. 


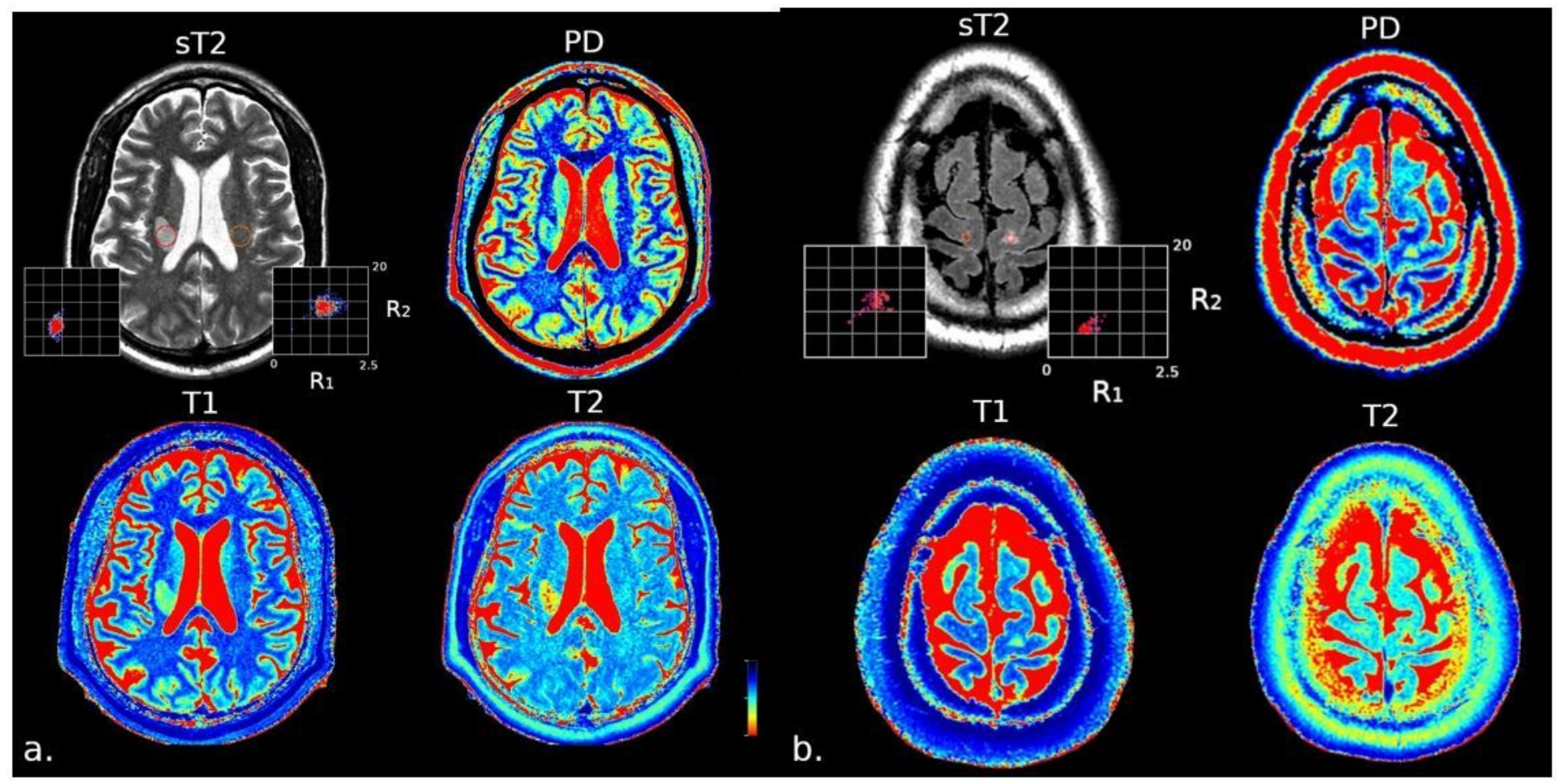

Figure 2

Representative examples of ROI in lesions (Fig. 2a: acute ischemic lesion DWI+/rADC, Fig 2b: chronic ischemic lesion WMH) and contralateral mirror NBR in a sT2 weighted image (upper left quadrant) with $\mathrm{R}_{1} \mathrm{R}_{2}$ Plot (scales: 0 to 2.5 [1/s]; 0 to 20 [1/s]) and synthetic T1 (lower left quadrant, red-to-blue colorbar scale: 0 to 2000 [ms]), PD (upper right quadrant, red-to-blue colorbar scale: 0 to 100 [pu]) and T2 (lower right quadrant, red-to-blue colorbar scale: 0 to 200 [ms]) maps.
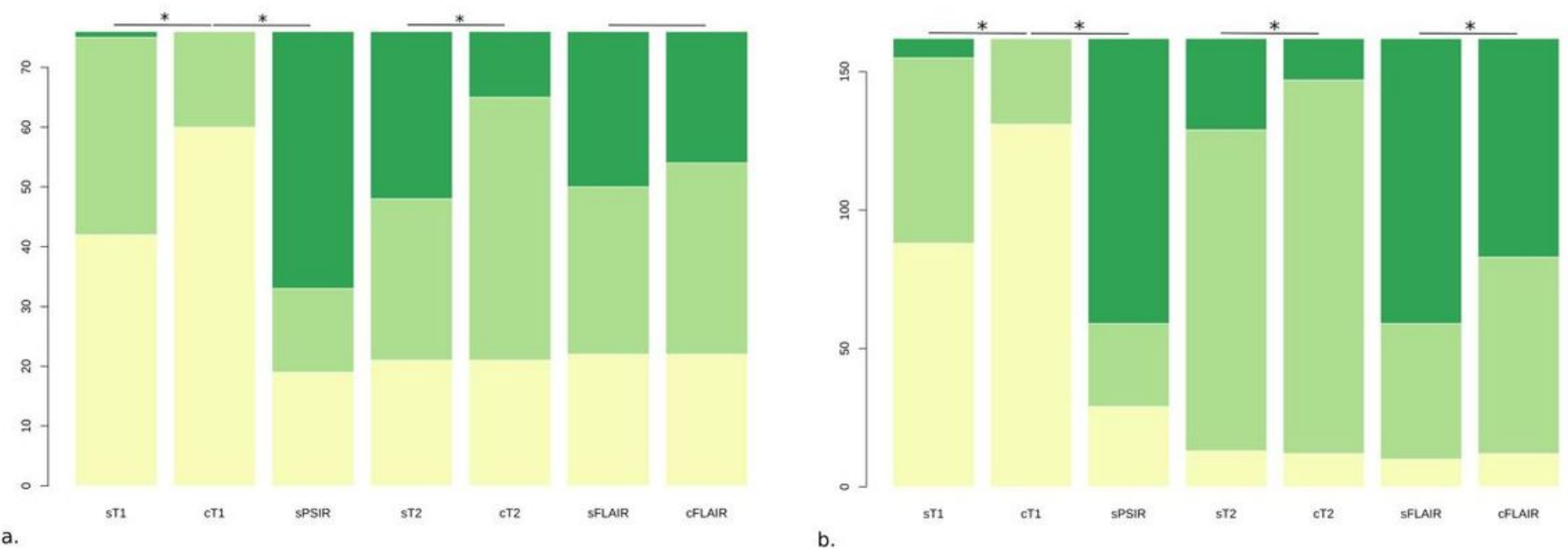

Figure 3 
Stacked bar plot of overall image quality for conventional and synthetic MR imaging for (Fig 3a) 46 acute (DWI+/rADC) and (Fig 3b) 90 chronic (WMH) ischemic lesions in the first 25 consecutive patients. Each contrast-weighted image quality for lesion definition and visibility was rated according to a 3-level score by two readers as: 1 - no lesion visible (yellow), 2 - lesion visible (light green), 3 - well-defined lesion (green). Significant differences $(P<.05)$ between weighted images are indicated by an asterisk.
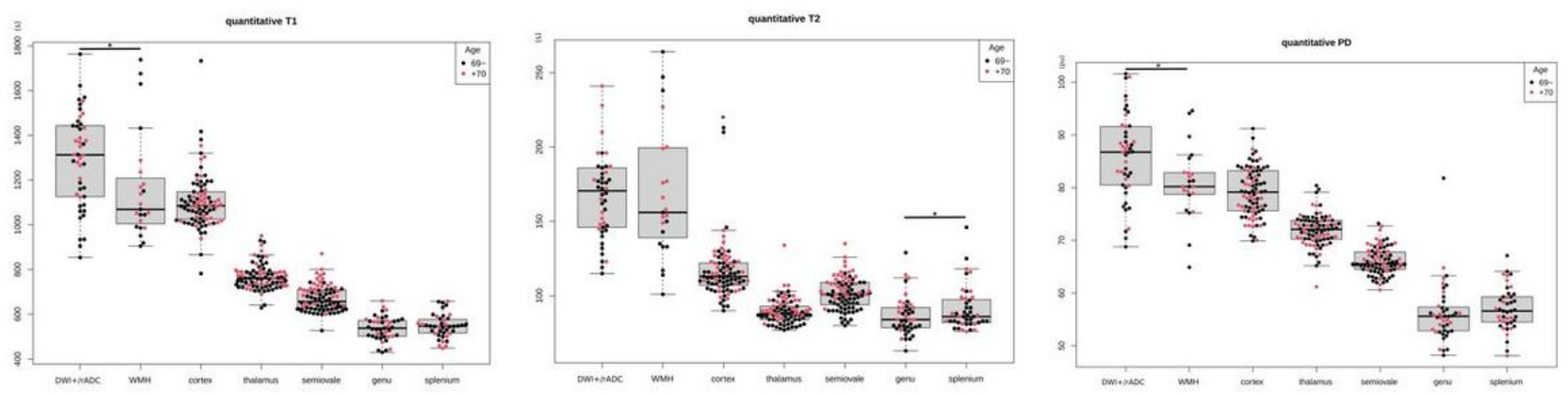

\section{Figure 4}

Beeswarm-box plots depicting quantitative synthetic findings of 46 acute (DWI+/rADC) and 23 chronic $(\mathrm{WMH})$ ischemic lesions, and, 430 NBR. Significant differences $(\mathrm{P}<.05)$ between ROI are indicated by an asterisk.

\section{Supplementary Files}

This is a list of supplementary files associated with this preprint. Click to download.

- tablesS1toS5.xlsx

- NameAffiliation.docx

- FigS1.jpeg

- FigS1withlegend.docx 